\title{
Spontaneous regression of squamous cell carcinoma arising from inverted papilloma: a case report
}

\author{
Chris Lee ${ }^{1}$, Sheeza Wajid ${ }^{1}$, Zao Yang ${ }^{1}$, Madeleine Samuelson ${ }^{2}$, Sanford Katz ${ }^{1}$, Michael Constantinescu ${ }^{3}$ and Lori Lemonnier ${ }^{*}$ \\ ${ }^{1}$ Department of Otolaryngology-Head and Neck Surgery, Louisiana State University Health, Shreveport, Louisiana, USA \\ ${ }^{2}$ Department of Otolaryngology-Head and Neck Surgery, Vanderbilt University Medical Center, Nashville, Tennessee, USA \\ ${ }^{3}$ Department of Pathology, Overton Brooks Veterans Affairs Medical Center, Shreveport, Louisiana, USA
}

\begin{abstract}
Background: Inverted papilloma is a benign, locally aggressive neoplasm arising from the nasal cavity or paranasal sinuses that is associated with carcinoma in $5-7 \%$ of cases. Spontaneous regression of malignant tumors is a well-documented but rare phenomenon. Yet, no case of spontaneous regression of a primary sinonasal malignancy has been reported to date. We present a case of spontaneous regression of squamous cell carcinoma arising from an inverted papilloma.
\end{abstract}

Methods: Case report and literature review.

Results: A 93-year-old male presented with persistent inverted papilloma with intracranial extension after previously failed surgical resection at an outside facility and subsequent treatment with radiation therapy. Sudden, rapid growth of the lesion into the orbit and skin of the medial canthus was biopsy-proven to be positive for invasive squamous cell carcinoma. Two months after entering hospice, the patient presented with no external, endoscopic, or radiographic evidence of the lesion, consistent with spontaneous regression. The patient expired several months later from complications secondary to cerebrovascular accident and was found to have no evidence of disease persistence or recurrence at that time.

Conclusion: Few cases of spontaneous regression of squamous cell carcinoma have been reported within the head and neck, with previous cases limited to primary locations within the oral cavity and oropharynx. To our knowledge, this is the first described case of spontaneous regression of squamous cell carcinoma of sinonasal origin.

\section{Introduction}

Inverted papilloma (IP) is a benign, locally-aggressive tumor of the nasal cavity or paranasal sinuses with potential for recurrence and malignant transformation. Both synchronous involvement with squamous cell carcinoma (SCC) and subsequent development of SCC at an IP site may occur. The rate of associated malignancy reported in recent literature is estimated at 5-15\% [1-3].

Spontaneous regression is a rare but recognized phenomenon, defined as the partial or complete disappearance of a malignant tumor in the absence of all treatment, or in the presence of therapy considered inadequate to exert significant influence on neoplastic disease, with the original presence of cancer proven by microscopy [4,5]. Current literature concludes that almost all types of malignant tumors can regress spontaneously, although some more frequently than others [4]. In the head and neck, spontaneous regression of SCC has previously only been reported to occur in the oral cavity and oropharynx [6,7]. We present a case of spontaneous regression of SCC of the paranasal sinuses arising from IP. To date this is the first case of spontaneous regression of a sinonasal malignancy reported in the English literature.

\section{Case presentation}

A 93 year old Caucasian male with history of IP presented to our institution for evaluation of a sinonasal mass with intracranial extension. The patient was first diagnosed with inverted papilloma eight years prior to presentation. The patient underwent two endoscopic sinus surgeries with resection of IP, including frontal sinusotomy, total ethmoidectomy and maxillary antrostomy, though both procedures concluded with known residual disease left along the cribriform plate. The second procedure was performed 17 months after the first. A third resection was planned but cancelled due to a myocardial infarction pre-operatively. Given his cardiac status and multiple other medical comorbidities, the residual disease was then treated with external beam radiation therapy. The patient was followed conservatively following this. At two and a half year follow up, the patient began complaining of severe headaches, and imaging was consistent with intracranial extension of persistent IP. At this point the patient was referred to our care.

The patient was presented at our institutional multidisciplinary head and neck tumor board, which recommended treatment with intensitymodulated radiation therapy (IMRT) targeting the intracranial portion of the lesion. At two month follow up after completion of IMRT, the patient presented with an ulcerative lesion of the left medial canthus associated orbital swelling (Figure 1). Biopsy of this mass revealed squamous cell carcinoma (Figure 2), with MRI demonstrating extension from the known sinonasal IP (Figure 3). The patient was

Correspondence to: Lori Lemonnier, Department of Otolaryngology-Head and Neck Surgery, Louisiana State University Health, Shreveport, 1501 Kings Hwy, Shreveport, LA 71103, USA, Tel: (318) 675-6262; Fax: (318) 675-6260; E-mail: llemo3@lsuhsc.edu

Key words: inverted papilloma, spontaneous neoplasm regression, squamous cell carcinoma, paranasal sinus neoplasms

Received: May 17, 2017; Accepted: June 07, 2017; Published: October 18, 2017 


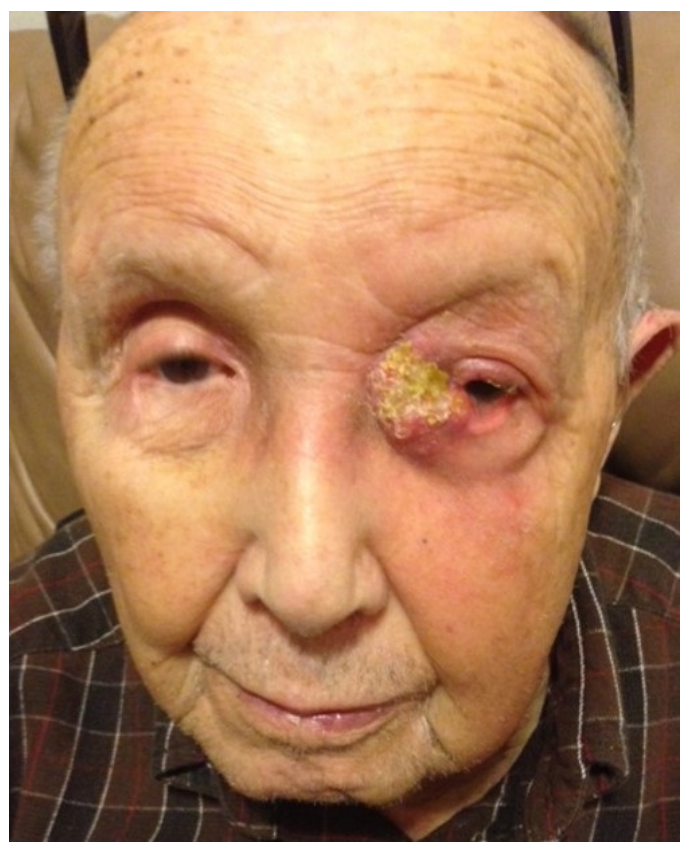

Figure 1. Medial canthal lesion, prior to biopsy.

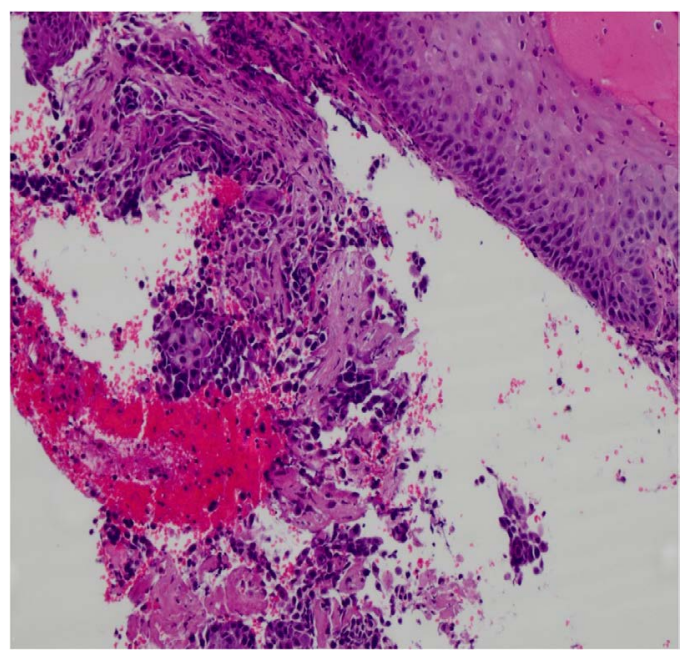

Figure 2. Histopathology, H\&E stain, medial canthal lesion.

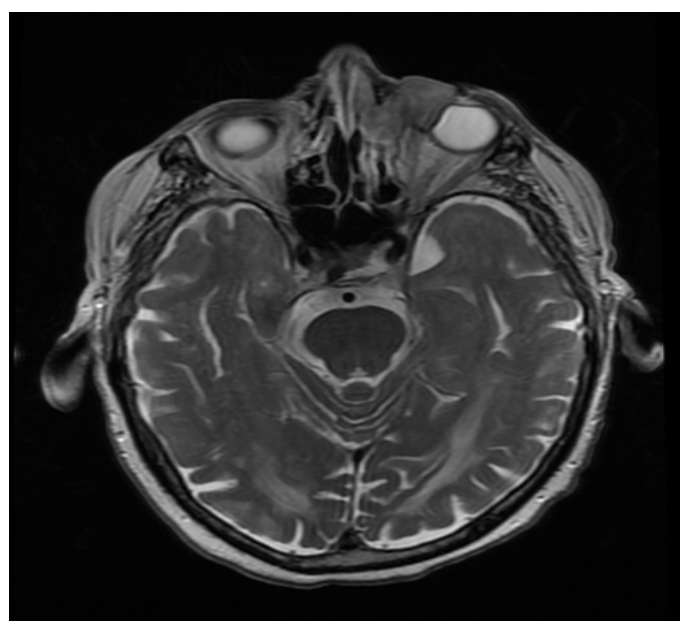

Figure 3. MRI obtained at the time of diagnosis. again discussed at our tumor board and deemed a poor candidate for all treatment modalities given his age, medical comorbidities, history of prior radiation, and the extent of the disease process. After discussion with the patient regarding the consensus opinion and the significant morbidity associated with the potential treatment options, he elected to enter hospice care.

Two months after recommendation for hospice, our patient was seen for routine follow up with spontaneous regression of the lesion on physical examination (Figure 4), nasal endoscopy and repeat imaging (Figure 5). He continued to do well until 5 months later when he expired from complications of a cerebrovascular accident. He demonstrated no evidence of recurrence at that time.

\section{Discussion}

Inverted papillomas are benign, locally aggressive tumors with potential for recurrence and malignant transformation [1]. IP accounts for $0.5-4 \%$ of all nasal neoplasms $[1,2]$. The most common site of origin is the lateral nasal wall; lesions involving the paranasal sinuses most frequently originate in the maxillary sinus, followed by the ethmoid sinuses [2]. The stage of an IP is most commonly reported using the Krouse classification and is based on tumor location, extent, and presence of malignancy [8]. IP most frequently presents in white males in their fifth to seventh decade with symptoms of unilateral nasal obstruction, nasal discharge, epistaxis, and facial pain and pressure, which can persist from months to years [9].

The etiology of IP has yet to be determined; however, the high prevalence of human papilloma virus (HPV) DNA detected in IP is suspected to be linked to its formation [10]. Existence of viral-like particles in IP tissue, evidence from clinical findings of multicentricity, and IP's high recurrence rate of 20-30\% support this theory [11-13]. IP demonstrates histological features also seen in exophytic papillomas, which frequently contain copies of HPV. HPV detection rates increase greatly in moderate to severe dysplasia and carcinomas that arise in IP [12]. Immunohistochemical staining patterns suggest an association of the p53 pathway in the malignant transformation of IP with higher levels of the $p 53$ gene in carcinoma specimens. However, the exact cause of growth, recurrence, and malignant transformation of IP remain unclear [14].

Definitive diagnosis is based on histopathologic analysis. There are three histological types of papillomas that occur in the sinonasal tract: exophytic, oncocytic and inverted papilloma. Inverted papillomas show downgrowth of the epithelium into underlying stroma resulting in an endophytic growth pattern by preservation of the epithelial basement membrane [13]. IP is commonly linked to various degrees of dysplasia, atypia, and squamous cell carcinoma [15]. Squamous cell carcinoma associated with IP can present either synchronously or metachronously [13]. Synchronous carcinoma occurs at a rate of $7 \%$ either from the papilloma or as a separate lesion; metachronous carcinoma develops at the site of the inverted papilloma in $3.6 \%$ of cases [1]. The rate of malignant association of inverted papilloma with squamous cell cancer is estimated at $5-15 \%$, with a rate of provable malignant transformation of $2-6 \%[13,14]$.

The primary treatment of inverted papilloma is complete surgical excision. Widespread training in and use of endoscopic techniques means that more nasal tumors can be resected via a less invasive, endoscopic approach [14]. Recurrence of IP may be the result of either incomplete removal of the lesion or development from predisposed mucosa [1]. There are no clear risk factors that predict risk of recurrence. 


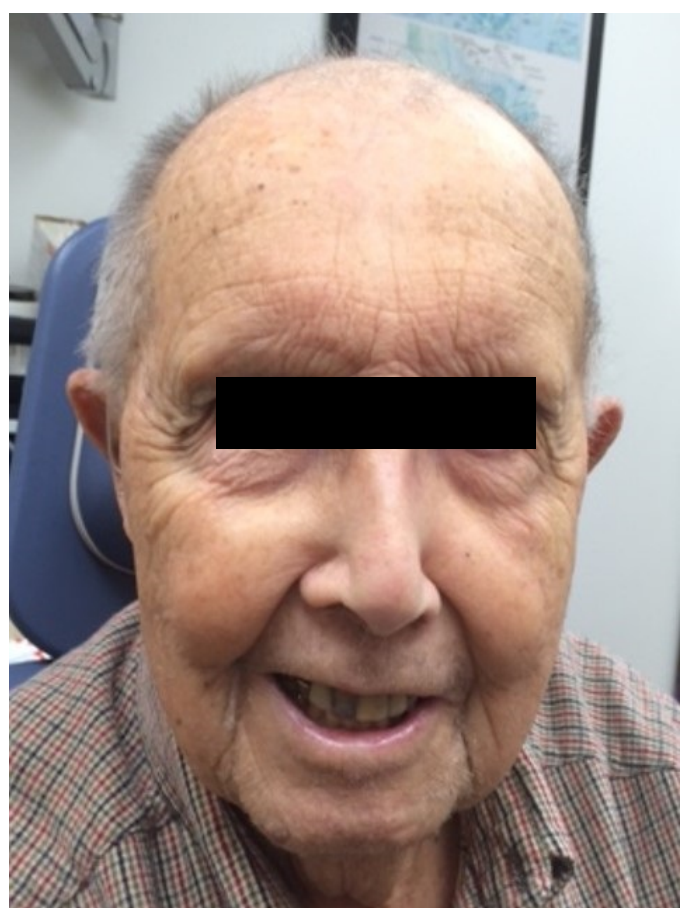

Figure 4. Spontaneous regression of SCC.

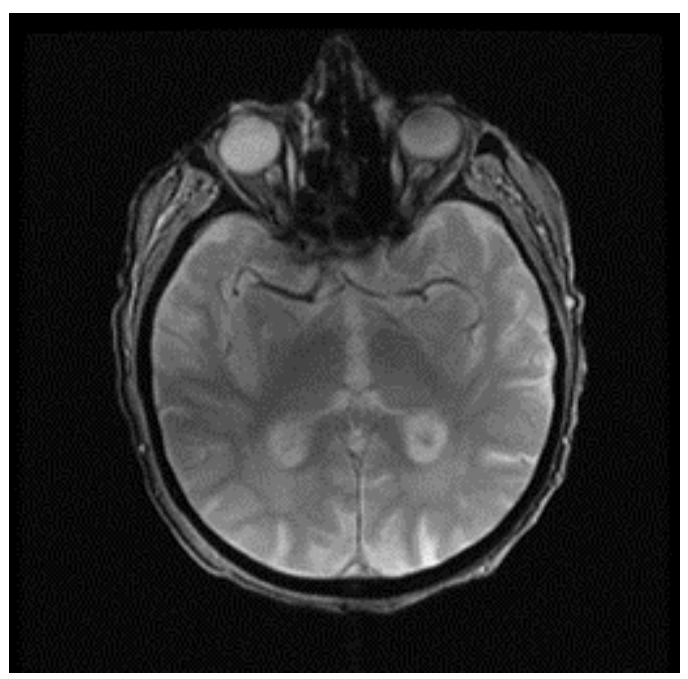

Figure 5. MRI 6 months after diagnosis.

Spontaneous regression is the partial or complete disappearance of a malignant tumor in the absence of all treatment, or in the presence of therapy considered inadequate to exert significant influence on neoplastic disease, with the original presence of cancer proven by microscopy $[4,5]$. Spontaneous regression is extremely rare, occurring in 1:80,000-1:140,000 of all reported malignant tumors [6]. The most commonly reported types include pediatric embryonal tumors, chorioepithelioma, renal adenocarcinoma, neuroblastoma, malignant melanoma, sarcomas, and carcinomas of the female breast, bladder and skin [7]. Previous cases of spontaneous regression of non-cutaneous head and neck malignancies have been limited to the oral cavity and the oropharynx [6,7]. Such cases include regression of oral lymphomas, oropharyngeal carcinomas and recurrent tongue carcinomas, as well as a novel case of a primary floor of mouth SCC [6]. An unusual case of diffuse large B cell Lymphoma (DLBCL), including a $2 \mathrm{~cm}$ tonsillar node, appeared with rapid growth after the patient suffered a broken arm, and was followed by $90 \%$ regression in the absence of chemotherapy agents [16]. At least 23 cases of spontaneous regression of Merkel cell carcinoma, a rare, cutaneous malignancy, have been reported to occur following incisional biopsy, including primary lesions of the nose and eyebrow $[17,18]$.

Causes for spontaneous regression remain unclear. Suspected contributing factors include the immune system, apoptosis, and conditions in the tumor microenvironment [4]. Regression of tumors has been associated with herbal medicine, surgical intervention, and vaccine therapy [19]. In addition, many reported cases of spontaneous regression are preceded by acute infection. Prior fever was recorded in $25-80 \%$ of documented cases of spontaneous regression in one study [5]. Infections associated with regression include gonorrhea, smallpox, malaria, influenza, in addition to other numerous pyogenic and nonpyogenic infections.6 Infection may trigger an increased host immune response, leading to an increased response against tumor cells [20]. Because of the wide diversity of organisms reported in spontaneous regression and the rapid rate at which regression typically occurs, it has been proposed that spontaneous regression is more likely to be linked to cell-mediated immunity rather than humoral immunity [5]. In this case it is possible that the incisional biopsy triggered an immune response that lead to spontaneous regression of the tumor.

\section{Conclusion}

Few cases of spontaneous regression of squamous cell carcinoma have been reported within the head and neck, with previous cases limited to primary locations within the oral cavity and oropharynx. To our knowledge, this is the first described case of spontaneous regression of squamous cell carcinoma of sinonasal origin. Though the exact causes of spontaneous regression are unclear, it is possible that the incisional biopsy of this tumor triggered an immune response leading to complete regression.

\section{References}

1. Mirza S, Bradley PJ, Acharya A, Stacey M, Jones NS (2007) Sinonasal inverted papillomas: recurrence, and synchronous and metachronous malignancy. J Laryngol Otol 121: 857-864. [Crossref]

2. Depondt J, Shabana el-H, Walker F, Pibouin L, Lezot F, et al. (2008) Nasal inverted papilloma expresses the muscle segment homeobox gene Msx2: possible prognostic implications. Hum Pathol 39: 350-358. [Crossref]

3. Busquets JM, Hwang PH (2006) Endoscopic resection of sinonasal inverted papilloma a meta-analysis. Otolaryngol Head Neck Surg 134: 476-482. [Crossref]

4. Ricci BS, Cerchiari UGO (2010) Spontaneous regression of malignant tumors: Importance of the immune system and other factors (Review). Oncol Lett 1: 941-946. [Crossref]

5. Thomas JA, Badini M (2011) The role of innate immunity in spontaneous regression of cancer. Indian J Cancer 48: 246-251. [Crossref]

6. de Andrade Sousa A, Lopes Rena R, Souza Silva G, Marcos Arantes Soares J, PorcaroSalles JM, et al. (2014) Spontaneous remission of a squamous cell carcinoma of the floor of the mouth. J Craniomaxillofac Surg 42: 1536-1539. [Crossref]

7. Thomas J (2011) Immunity over inability: The spontaneous regression of cancer. J Nat Sci Biol Med 2: 43-49. [Crossref]

8. Krouse JH (2000) Development of a staging system for inverted papilloma Laryngoscope 110: 965-968. [Crossref]

9. Karkos PD, Fyrmpas G, Carrie SC, Swift AC (2006) Endoscopic versus open surgical interventions for inverted nasal papilloma:a systematic review. Clin Otolaryngol 31: 499-503. [Crossref]

10. Georg E, Mühling J, Hassfeld S (2007) Inverted papilloma of paranasal sinuses. $J$ Craniomaxillofac Surg 35: 21-29. 
11. Thorp MA, Oyarzabal-Amigo MF, du Plessis JH, Sellars SL (2001) Inverted papilloma: a review of 53 cases. Laryngoscope 111: 1401-1405. [Crossref]

12. Lawson W, Schlecht NF, Brandwein-Gensler M (2008) The role of the human papillomavirus in the pathogenesis of Schneiderian inverted papillomas: an analytic overview of the evidence. Head Neck Pathol 2: 49-59. [Crossref]

13. Sandison A (2009) Common head and neck cases in our consultation referrals: diagnostic dilemmas in inverted papilloma. Head Neck Pathol 3: 260-262. [Crossref]

14. Govindaraj S, Wang H (2014) Does human papilloma virus play a role in sinonasal inverted papilloma? Curr Opin Otolaryngol Head Neck Surg 22: 47-51. [Crossref]

15. von Buchwald C, Bradley PJ (2007) Risks of malignancy in inverted papilloma of the nose and paranasal sinuses. Curr Opin Otolaryngol Head Neck Surg 15: 95-98. [Crossref]
16. Engel PA, Lee C (2009) Sudden appearance and spontaneous regression of diffuse large B cell lymphoma in a man with a broken arm. BMJ Case Rep 2009.

17. Richetta AG, Mancini M, Torroni A, Lorè B, Iannetti G, et al. (2008) Total spontaneous regression of advanced Merkel cell carcinoma after biopsy: review and a new case. Dermatol Surg 34: 815-822. [Crossref]

18. Pang C, Sharma D, Sankar T (2015) Spontaneous regression of Merkel cell carcinoma: A case report and review of the literature. Int J Surg Case Rep 7: 104-108. [Crossref]

19. Choi N, Cho JK, Baek CH, Ko YH, Jeong HS (2014) Spontaneous regression of metastatic cancer cells in the lymph node: a case report. BMC Res Notes 7: 1. [Crossref]

20. Kalialis LV, Drzewiecki KT, Klyver H (2009) Spontaneous regression of metastases from melanoma: review of the literature. Melanoma Res 19: 275-282. [Crossref]

Copyright: $(2017$ Lee C. This is an open-access article distributed under the terms of the Creative Commons Attribution License, which permits unrestricted use, distribution, and reproduction in any medium, provided the original author and source are credited. 